\title{
Identification of molecular targets for mycotoxins related to autism development
}

\author{
A. Marabotti ${ }^{凶}$, M. Landini', A. Mezzelani', L. Milanesi', M.E. Raggi ${ }^{2}$ \\ "Istituto di Tecnologie Biomediche, CNR, Segrate, Italy \\ 2IRCCS "E.Medea" Associazione "La Nostra Famiglia", Bosisio Parini, Italy
}

\section{Motivations}

Autistic spectrum disorder (ASD) is an ensemble of developmental disorders impacting on the social relationships, the development of the language and of interpersonal communications and the behaviour of affected people. A number of factors have been supposed to cause ASD, including a direct genetic component (accounting for less than $10 \%$ of cases), epigenetics and environmental factors such as pollutants, heavy metals, viruses and vaccines. To date, however, none of these factors have been definitely associated to ASD. Further studies hypothesized the presence of "vulnerability genes" whose variants are not causative of autism per se, but can increase the susceptivity of the individuals towards several factors, ultimately leading to autism development. In people with ASD, gastrointestinal (GI) disorders are commonly reported. The "leaky gut hypothesis" theory speculates that impaired gut permeability permits the entry of molecules such as toxins in the bloodstream, both affecting directly the central nervous system (CNS), and causing sensitization of the gut mucosal immune system. Mycotoxins are food contaminants present ubiquitously and derived from the secondary metabolism of several moulds and fungi. The consumption of several foods, especially those derived from cereals and milk, exposes people to the intake of high level of mycotoxins. Children can be more exposed than adults to mycotoxins, for their particular diet, for a lower diet variability with respect to adults, and for the fact that a newborn assumes more food than an adult, in proportion to his/her body weight. Once introduced in the body, mycotoxins could promote sneaky negative effects in the Gl trait and in other tissues, playing a role in the development of syndromes of unknown etiology. In our work, we used bioinformatics approaches to find potential targets for the binding of mycotoxins, in order to verify the existence of a possible role in ASD onset, and we tested a small sample of ASD patients and controls to verify the alteration of genes identified as potential targets.

\section{Methods}

The Web server TarFisDock was used to identify proteins able to bind four selected mycotoxins: ochratoxin, gliotoxin and fumonisins B1 and B2, with a reverse docking approach. The 3D structures of these molecules were obtained in .sdf format from PubChem. Both neutral and charged formes were taken into account. Targets were searched among the 3D structures of proteins deposited in PDTD database associated to TarFisDock server. Analyses of the involvement of these targets in different pathway networks were made using KEGG database. The further docking studies were made using AutoDock v. 1.5.2. and ADT. A sample of 52 ASD patients and 40 healthy parental and uncorrelated controls were analysed to search for the presence of mutations in selected genes among the targets identified by reverse docking approaches. For each patient, DNA was isolated from peripheral blood and NLGN3 and NLGN4X exons were amplified and sequenced by PCR and Sanger method, respectively.

\section{Results}

The reverse docking approach identified several proteins that are involved in pathways related to CNS development and/or pathologies, as possible targets for the binding of each mycotoxin. Moreover, the relative abundance of proteins related to CNS pathways is increased with respect to the statistical distribution of targets among all the classes identified by the tool. Several proteins in common to all these mycotoxins were identified among the top $10 \%$ of all possible targets. In particular, the structural class of cholinesterase was found to be a common target for mycotoxins. This is very interesting, since neuroligins, proteins of the cholinesterase structural class, are directly associated in literature with autism. We performed traditional docking simulations to verify if mycotoxins can bind to these proteins. Results 
confirmed the possibility of some mycotoxins to bind to neuroligins, in particular neuroligin 3 and 4, associated to chromosome $X$ and therefore very interesting, given the ratio of 4:1 between males and females for ASD onset. Moreover, with docking studies, we were able to find that some mycotoxins, in neuroligin $4 \mathrm{X}$, are predicted to interact directly with residues whose mutation is related to autism. This suggests that indeed the mutation of this protein can modulate its ability to interact with exogenous toxins, and therefore people carrying these mutations could be more susceptible to their negative effects. The genetic analysis conducted on selected autistic patients and controls confirmed the presence of SNP and mutations on the exons of NLGN3 and NLGN4X. Further studies will be made to establish a statistical relationship among the presence of mutations and the distribution of mycotoxins into the bodily fluids of these people.

\section{Acknowledgements}

Ricerca Finalizzata e Giovani Ricercatori 2009 (GR-2009-1570296), FIRB-MIUR Italbionet (RBPRO5ZK2Z and RBIN064YAT_ 003), Flagship Project InterOmics. 